\title{
AUTOMATIC LICENSE PLATE LOCALIZATION USING DIFFERENT WAVELET TRANSFORMS
}

\author{
X.Ascar Davix ${ }^{1,}{ }^{*}$,C.Seldev Christopher ${ }^{2}$ and P.CAROLIN REEBA ${ }^{3}$ \\ ${ }^{1}$ Assistant Professor, Department of Electronics and Communication Engineering, \\ St.Xavier’s Catholic College of Engineering, Anna University, India. Email : davixascar@gmail.com \\ ${ }^{2}$ Professor, Department of Computer Science Engineering, \\ St.Xavier's Catholic College of Engineering, Anna University, India \\ ${ }^{3}$ PG student, St.Xavier’s Catholic College of Engineering, Anna University, India \\ ${ }^{*}$ Corresponding author
}

\begin{abstract}
Automatic license plate recognition (ALPR) is a technique that extracts the vehicle license plate information from an image or a sequence of images. License Plate Recognition is used increasingly nowadays for automatic toll collection, maintaining traffic activities, law enforcement and parking. The basic step in License Plate detection is the localization of number plate. In this paper a new method based on different wavelet transform and empirical mode decomposition (EMD) analysis is used to search for the location of a license plate in an image. Haar and Daubechies wavelet are used to locate the license plate. Thus a comparison is made by analyzing the performance of these wavelets. Haar wavelet can detect various types of license plates including different countries, different colors, different character types and perspective distortion, dirty or blurry images. Similarly, Daubechies wavelet can also locate plates with different shapes, sizes, and colors under varying lighting conditions and distances.
\end{abstract}

Keywords-recognition; wavelet transform; image details; localization; license plate

\section{INTRODUCTION}

License plate recognition (LPR) plays a significant role in many applications relevant to transportation systems such as traffic management and analysis, speed control, automobile theft prevention, parking lot management, and many other research areas. For any proposed LPR system, the effect of variable illumination, complicated backgrounds, and the distortive effect of vehicle speed on license plates are some of the main problems which have to overcome. This paper is organized as follows: An LPR method, which uses various features, is studied in section 2. An automatic license plate localization system using Haar and Daubechies wavelet is studied in section 3. In section 4, the results of both the system are explored. A conclusion of the paper is given in section 5 .

\section{PREVIOUS WORK}

All LPL techniques locate the license plate by analyzing the features of license plates. Some of the features that are considered for analysis are color, shape, texture, presence of characters. Two or more features can be combined to identify the location of the license plate.

\section{A. License Plate Extraction Using Boundary/Edge Information}

In [1] the edge based methods pertaining to plate localization are based on the principle that the plates appearing in the vehicle image have some distinct features that make it distinguishable easily from the rest of the image such as high contrast of license plate as compared to the rest of the image of the vehicle. In [2] edges are extracted and geometrical features are analyzed to detect valid license plate region. In [3], a new approach of edge enhancement for enhancing detection of License Plate after improving the contrast by using Contrast Limited Adaptive Histogram Equalization is proposed.

\section{B. License Plate Extraction Using Texture Features}

In [7], the pulse coupled neural network (PCNN) is presented for image segmentation. All the methods based on texture have the advantage of detecting the license plate even if its boundary is deformed.

\section{License Plate Extraction Using Color Features}

In [5], a color-based LPL method is presented. This consists of three modules: color edge extraction, de noising and searching. Color edge extraction, is designed by color-discrete characteristics of license plates in the trichromatic wavebands. In [4], a new algorithm of vehicle license locating based on color segmentation is proposed. 


\section{License Plate Extraction Using Character Features}

In [6], a two-stage segmentation method is proposed. At the first stage, a novel template matching method is presented using a harrow-shaped filter (HSF) bank and minimum response. This method does not guarantee that the segmented characters are perfect for recognition.

From the review above it is seen that some methods deals with still images while some can detect only limited style of license plates. Even though some methods show high accuracy they are time consuming and complicated.

\section{A. License Plate Features}

\section{A NOVEL LPR METHOD}

The license plate has the factors, such as rotation angle, number of character lines, character types and formats can strongly change the style of license plates. The registration plate consists of 4 parts, they are:

- The first two letters represent the state or Union Territory where the vehicle is registered.

- The next two digit numbers indicate the sequential number of a district.

- The third part is a four digit number unique to each plate.

- The fourth part is an international oval "IND" and the top it a Hologram having a Chakra.

B. Localization using Haar wavelet

The block diagram of localization using Haar wavelet is shown in

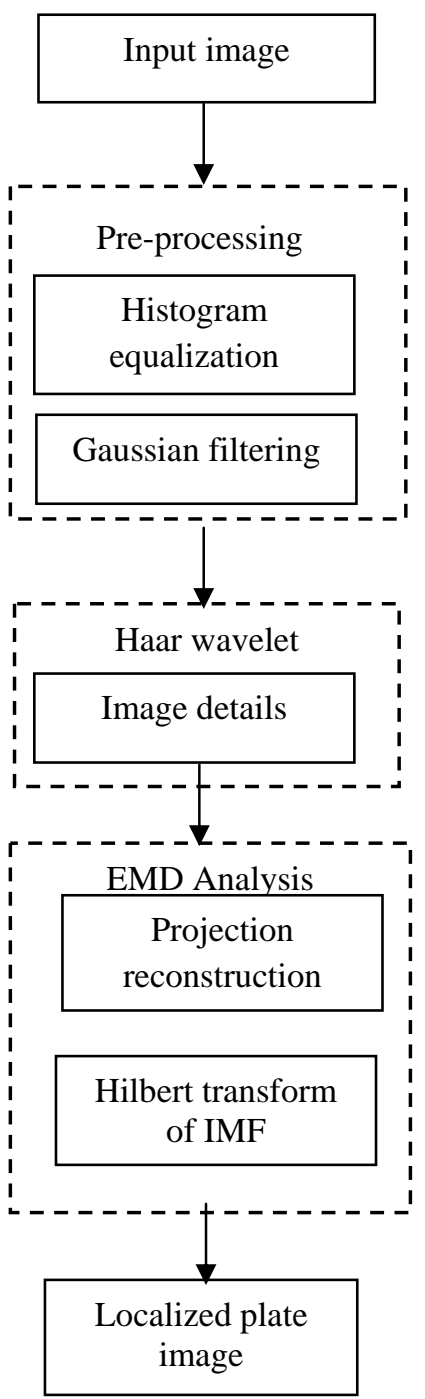

Fig. 1.Block diagram of localization using haar wavelet.

The input image is generally a color image taken by a camera. The camera may be a low resolution camera. Also the image may be affected by the environmental variations. The image captured strongly depends on the hardware used and environment conditions. As a result, a graphic file is obtained. 
1) Preprocessing: To reduce error, some preprocessing is needed. Preprocessing is composed of the following two steps.

a) Gaussian filtering: Images taken from cameras may suffer from noise interference, hence filters like Gaussian filter, median filter, etc. are used. Gaussian filter has rotational symmetry, so it can filter noise in all direction.

b) Histogram equalization: The input image to be enhanced has continuous gray values, with black values represented as $r=0$ and white values as $r=1$. Based on the histogram of the input image, design a gray value transformation $s=T(r)$. This will enhance the image. The output image will have all gray values in "equal proportion”.

2) Wavelet Transform: The process of a two dimensional wavelet transformation to an image is shown in Fig. 2. Here Haar wavelet is used. $\mathrm{G}$ stands for a low pass filter while $\mathrm{H}$ is a high pass filter. When the input image $A_{j}$ undergoes Haar wavelet transform, the image is decomposed into 4 components, i.e., approximation image $A_{j-1}$, horizontal details $H_{j-1}$, vertical details $V_{j-1}$ and diagonal details $D_{j-1}$.

Take $H_{j-1}$ as an example, the input image is first dealt by a low pass filter, so that low frequencies in the vertical direction are mainly kept. Then the processed image is dealt by a high pass filter which allows high frequencies in the horizontal direction retain. As a result, horizontal details are enhanced and vertical details are smoothed.
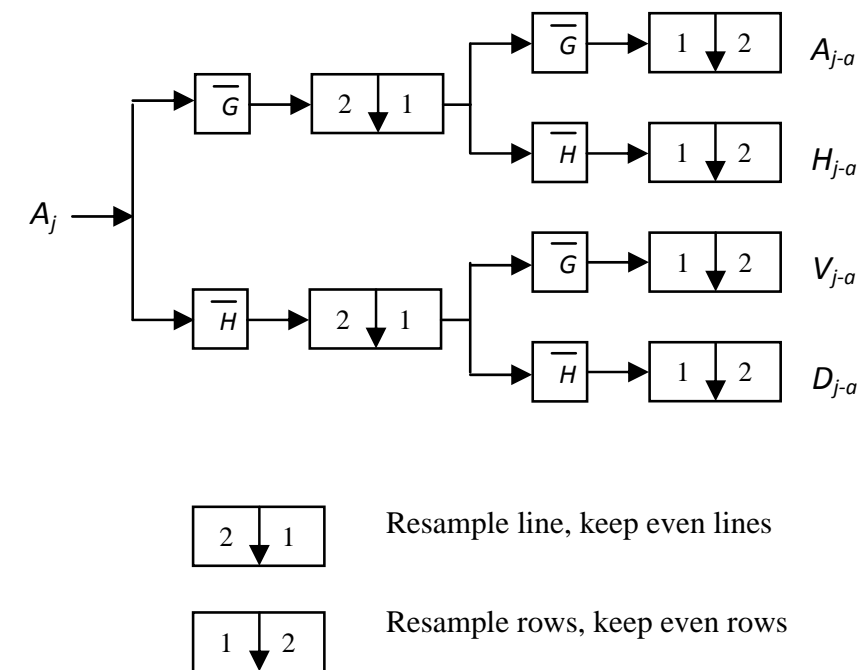

Fig. 2 Wavelet transform of images.

Similarly, vertical details are also generated. Wavelet transform can separate the detail information of the license plate from the whole vehicle images. Hence the main information related to the license plate will not be lost.

Then project the vertical details onto the $\mathrm{Y}$ axis, a wave crest will be generated by the character details since all the characters lie in a same horizontal line. A wave crest in the projection dataset can be located, and the $\mathrm{Y}$ position of the license plate will be easily detected. Similarly, project the horizontal details onto the $\mathrm{X}$ axis and a wave crest is generated and the $\mathrm{X}$ position of the license plate will be detected.

3) EMD Analysis: EMD is a data-driven time-frequency technique that decomposes a signal into a finite set of components called intrinsic mode functions (IMFs). It is a useful tool in extracting physically meaningful information. These components represent the local different oscillation modes that occur in the original dataset. IMFs can be obtained from an iterative sifting process. This stops when the number of extrema reaches a stop criterion. This method is used for analyzing the nonlinear and non-stationary projection data.

a) Sifting Algorithm: The EMD algorithm decomposes the signal $x(t)$ as

$$
x(t)=\sum_{j=1}^{n} c_{j}(t)+r_{n}(t)
$$

where $c_{j}(t)$ represents the IMFs and $r_{n}(t)$ is the residual.

The sifting process algorithm is as follows:

Step 1:

$$
\text { Let } r_{0}(t)=x(t) \text { and } j=1
$$

Step 2:

Extract the $\mathrm{j}^{\text {th }}$ IMF component 
i. $\quad$ Let $h_{0}(t)=r_{j-1}(t)$ and $i=1$.

ii. Identify all local maxima and minima of $\mathrm{h}_{\mathrm{i}-1}(\mathrm{t})$.

iii. Find the upper envelope $e_{\max }(t)$ and lower envelopes $e_{\min }(t)$ that interpolate all the maxima and minima respectively.

iv. Calculate the envelope mean $m_{i-1}(t)=1 / 2\left[e_{\max }(t)+e_{\min }(t)\right]$

v. $\quad h_{i}(t)=h_{i-1}(t)-m_{i-1}(t), i=i+1$

vi. Repeat (ii-v) until $h_{i}(t)$ becomes an IMF. Then let $c_{j}(t)=h_{i}(t)$

$\underline{\text { Step 3: }}$

$$
r_{j}(t)=r_{j-1}(t)-c_{j}(t), j=j+1
$$

Step 4:

Repeat (2-3) until $r_{\mathrm{j}}(\mathrm{t})$ matches the stopping criterion.

4) Projection Reconstruction: After getting the projection of the values of all the pixels onto the $Y$ axis, the EMD analysis is applied. The original dataset is decomposed into several IMF components. The projection is decomposed into various frequency bands from high to low. IMF1 shows the highest frequency component. At the same time, for IMF1 the amplitude and the standard deviation is low. It implies that the stochastic noise may be contained in IMF1. Similarly, the remaining IMFs may contain other higher frequency details.

To find the vertical location of a license plate, beside the $\mathrm{Y}$ position of the located maximum find the two minima in the reconstructed projection, which indicates the upper and lower boundaries of a license plate. If they all fail to detect the license plate, it is considered that no license plate exists in the processed image.

After the $\mathrm{Y}$ position of the license plate is located, the projection of the horizontal details onto the $\mathrm{X}$ axis will be decomposed by EMD analysis and the X position of a license plate is detected in a similar way.

C. Localization using Daubechies wavelet

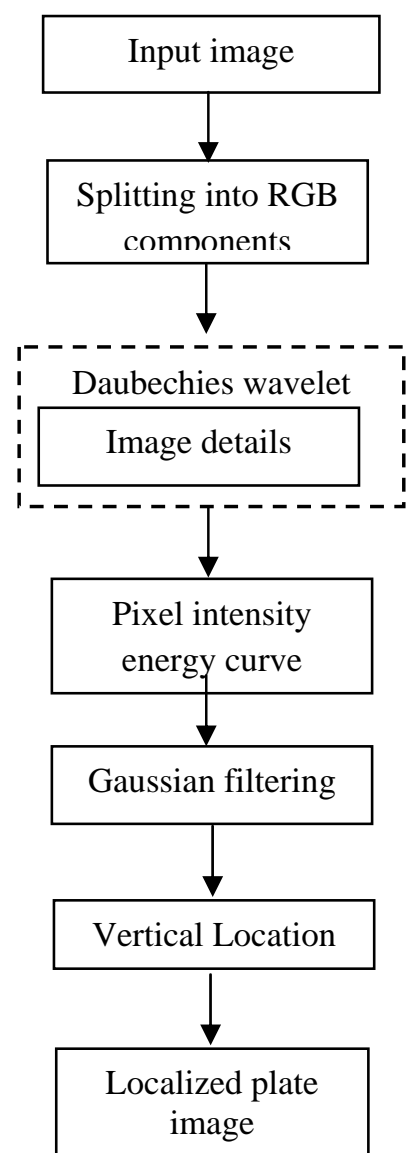

Fig. 3 Block diagram of localization using Daubechies wavelet 
The block diagram of localization using Daubechies wavelet is shown in Fig. 3. The image of the vehicle is split to the RGB components. $A R, A G$, and $A B$ are the RGB spectrum Here, $A B$ is taken as the candidate for plate localization and wavelet decomposition is performed to compute the approximation and details coefficients i.e., horizontal, vertical and diagonal details. The horizontal and vertical frequency energies can locate the license plate because the plate has a high frequency.

To find vertical location of plate, we plot the pixel intensity's energy curve for each row of the vertical frequency band. Similarly, to find the horizontal location, plot the pixel intensity's energy curve for each column of the horizontal frequency band. Then Gaussian filter is applied on both energy curves. The vertical frequency curve's high energies represent the location of number plate because, a higher frequency is found near the plate.

To compute the high-energy frequency band, compute a threshold value to operate on the vertical and horizontal frequency energy curves. Then take half of the maximum vertical energy and move from left to right and right to left in the vertical frequency curve to compute the $y$ coordinates. The $y$ coordinates that are greater than the threshold value represent the plate's vertical location.

Similarly, to find the horizontal location repeat the process as we did to find it vertically. In order to compute the threshold value 0.9 times the maximum $y$ coordinates can also be used and traverse from left to right and right to left to compute the $x$ coordinates. In practical, some adjustments might be required to compute the threshold values for horizontal and vertical locations.

\section{RESULTS AND DISCUSSION}

\section{A. Localization using haar wavelet}

The input image is shown in Fig. 4

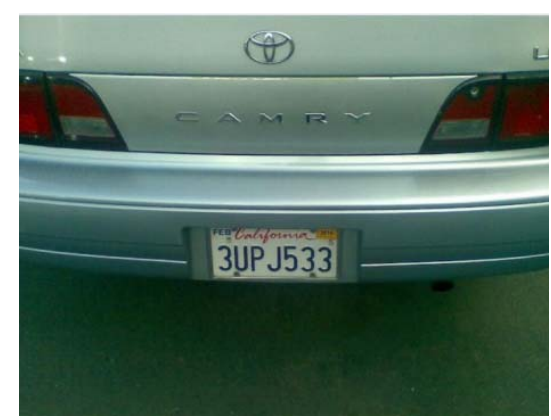

Fig. 4 Input image

Initially preprocessing is done on the input image. Preprocessing involves two steps Gaussian filtering and histogram equalization. Gaussian Filter blurs the image. reduce the noise and the image details. Histogram equalization is a technique is used to enhance contrast. The equalized image and its histogram are shown in Fig. 5.

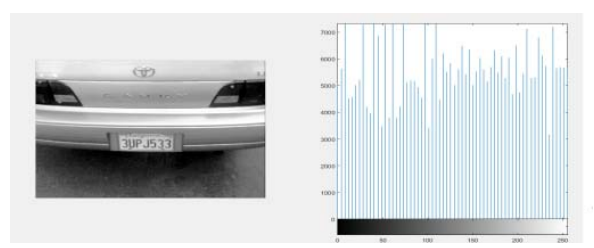

Fig. 5 Equalized image and its histogram

Then haar wavelet transform is applied, hence the image is decomposed into 4 components, vertical detail, horizontal detail, and diagonal detail, and approximation image. Approximation includes information about the global properties of analyzed image. Horizontal details include information about the vertical lines hidden in image. Vertical details contain information about the horizontal lines hidden in image. Diagonal details embrace information about the diagonal details hidden in image. The below Fig. 6 shows the 4 components. 

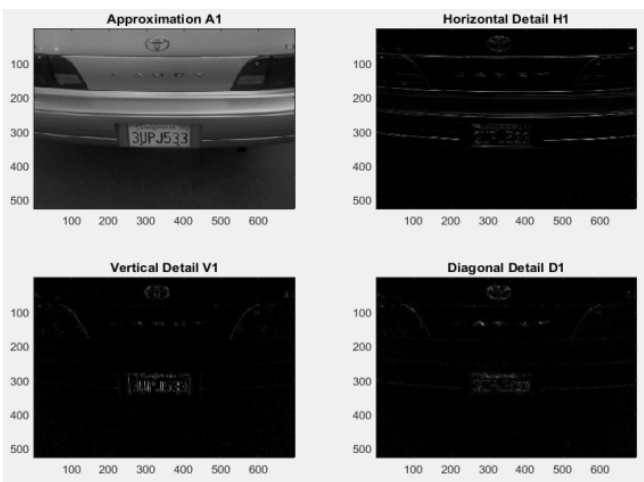

Fig. 6 Result of haar wavelet transform

When projecting the vertical details onto the $\mathrm{Y}$ axis, the entire characters lie in a same horizontal line so there will be a wave crest generated by the character details. Similarly, when projecting the horizontal details onto the $\mathrm{X}$ axis, there will be a wave crest generated by the character details.

The projection of the vertical details onto the $\mathrm{Y}$ axis and projection of the horizontal details onto the $\mathrm{X}$ axis is shown in Fig. 7 and Fig. 8.

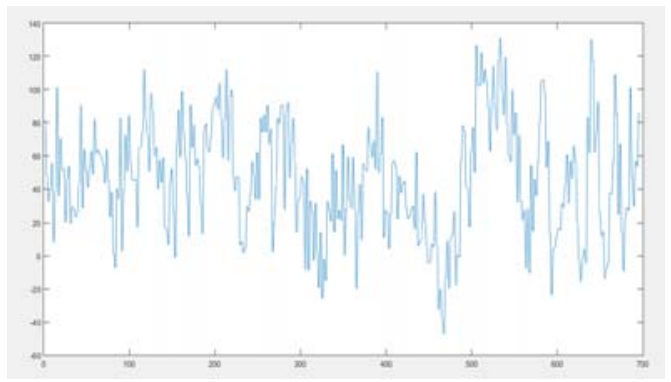

Fig. 7 Projection of the vertical details onto the $\mathrm{Y}$ axis

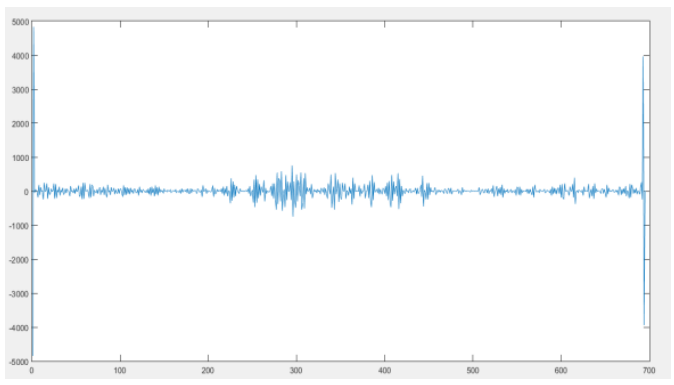

Fig. 8 Projection of the horizontal details onto the $\mathrm{X}$ axis

The projection of the vertical details onto the $\mathrm{Y}$ axis and projection the horizontal details onto the $\mathrm{X}$ axis is decomposed by EMD analysis. Thus the data is decomposed into a finite set of components called intrinsic mode functions (IMFs). In this project, the original dataset is decomposed into eight IMF components. The result of EMD analysis is shown in Fig. 9 and Fig. 10.

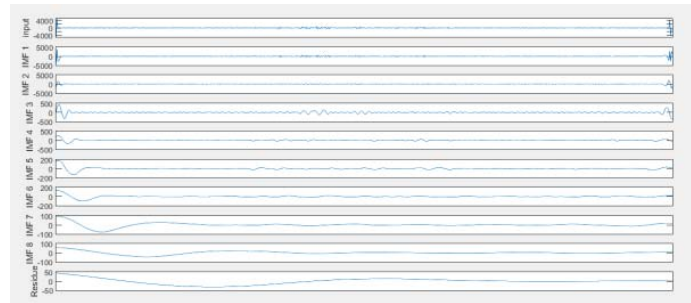

Fig. 9 IMFs and residual of the projection of the vertical details of Fig 5 onto the Y axis 


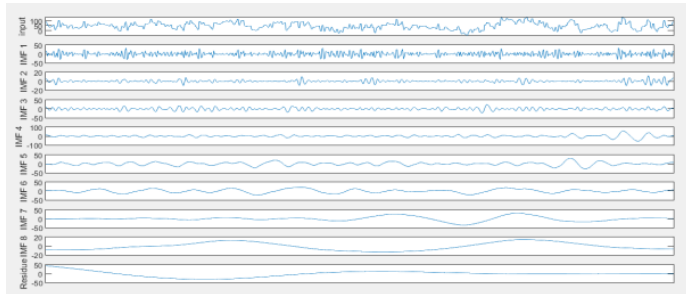

Fig. 10 IMFs and residual of the projection of the horizontal details of Fig. 6 onto the $\mathrm{X}$ axis

Then ignore the first several $(n / 2$ or $n / 2+1, \mathrm{n}$ is the number of the IMFs) higher frequency components of the decomposed IMFs, the projection can be reconstructed by the remaining IMFs and the contour of the projection will be kept. The final located license plate is shown in Fig. 11.

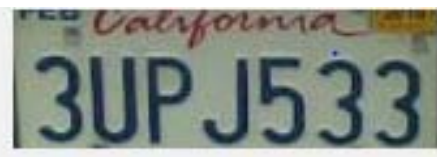

Fig. 11 Localized license plate

\section{B. Localization using haar wavelet}

The captured image is splitted into RGB components. The RGB components of the original image are shown in Fig. 12.

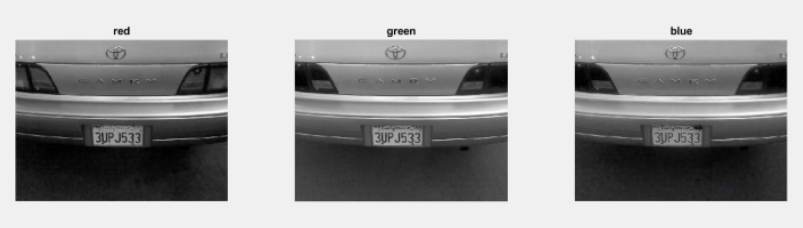

Fig. 12 RGB components of image

Then Daubechies wavelet transform is applied, hence the image is decomposed into 4 components, vertical detail, horizontal detail, and diagonal detail and approximation image. The below Fig. 13shows the 4 components.
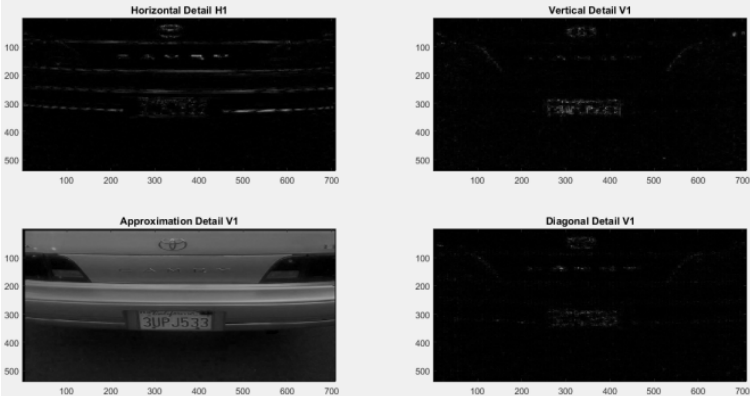

Fig. 13 Result of Daubechies wavelet transform

The horizontal and vertical frequency energy curves are plotted. The Vertical frequency energy curves is plotted by summing up pixel intensities of each row for vertical location and horizontal frequency energy curves is plotted by summing up pixel intensities of each column for horizontal location. The horizontal and vertical frequency energy curves are shown in Fig. 14 and Fig. 15.

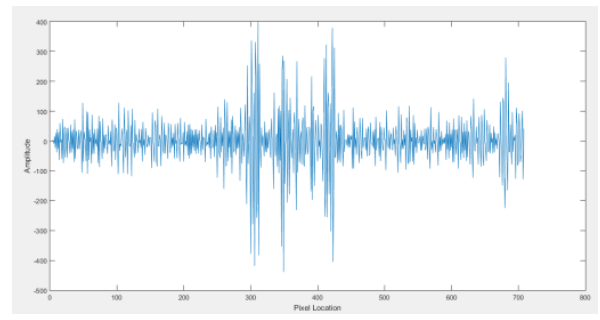

Fig. 14 Horizontal frequency energy curve 


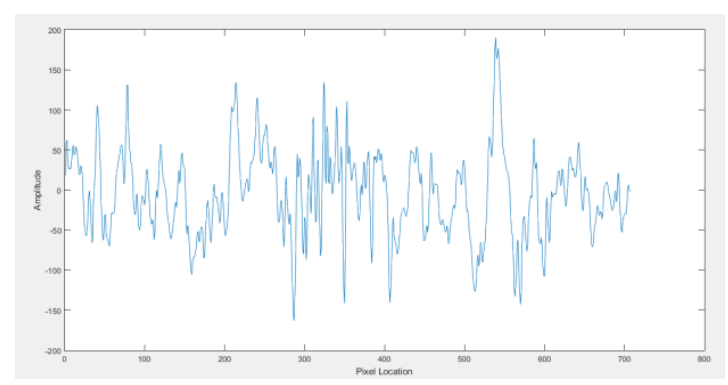

Fig. 15 Vertical frequency energy curve

Gaussian filter is applied on both the energy curves to smooth them. The high energy in the curve represents the location of the license plate.The result of applying Gaussian filter is shown in Fig. 16 and Fig. 17.

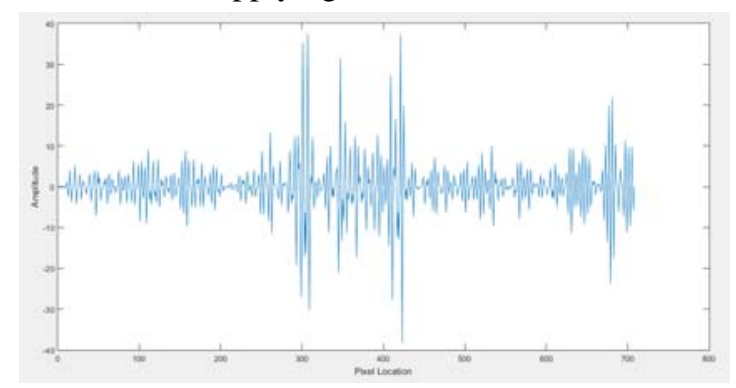

Fig. 16 Smoothed horizontal frequency energy curve

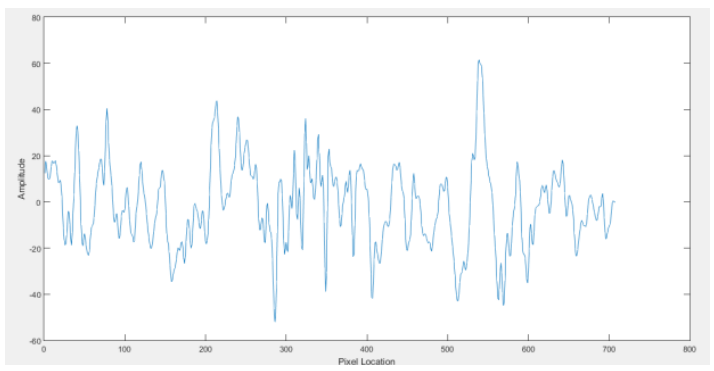

Fig. 17 Smoothed vertical frequency energy curve

Then calculate a threshold value to operate on both horizontal and vertical energy curve. Take half the maximum vertical energy and move from left to right and right to left in the vertical frequency curve to compute the $y$ coordinates. The $y$ coordinates that are greater than the threshold value represent the plate's vertical location. The same procedure is repeated to find the horizontal location. The location of the license plate is shown in below Fig. 18.

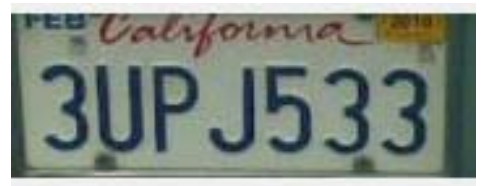

Fig. 18 Localized license plate

\section{Performance Analysis}

The accuracy of both the methods are analyzed with a database consisting of about 500 images. The database consists of the normal images acquired on the road, images obtained under various illumination scenarios which include daylight, night and shadow. The illumination not only changes slowly as daytime progresses but may change rapidly due to weather conditions and also includes the images obtained with complex backgrounds.

Using Haar wavelet of the 500 images, 462 images are correctly located and 34 images failed to be located. The localization failed for tilted images, blurred images. Also if there are other objects in the background the detection may not be effective. The location of the license plate number is not absolute in a vehicle picture which consists of abundant complex non-license plate information.

Using Daubechies wavelet of the 500 images, 485 images are correctly located and 15 images failed to be located. The localization failed for skewed and distorted images. The correctly located images and the accuracy of both the methods are listed in Table I. 
TABLE I PERFORMANCE COMPARISON OF HAAR AND DAUBECHIES WAVELET

\begin{tabular}{|l|l|l|}
\hline & Haar wavelet & Daubechies wavelet \\
\hline Correctly located images & 462 & 480 \\
\hline Accuracy(\%) & 92 & 96 \\
\hline
\end{tabular}

\section{CONCLUSION}

License Plate Localization is an image processing technology used to locate a license plate in an image. This paper shows the comparison made between two localization methods using Haar and Daubechies wavelet. Comparison of two methods in terms of their accuracy, conditions where they failed to locate the license plate is addressed. Haar wavelet can locate the license plate accurately without any change in the speed of the whole system. The accuracy of localization of this method is $92 \%$. Daubechies wavelet can locate the license plate under all weather conditions. The accuracy of localization of this method is $96 \%$. The future research will be focused in the recognition of characters.

\section{REFERENCES}

[1] Amir H.A., Md. Jan N. and Mahmood F, ‘An Iranian License Plate Recognition System Based on Color Features’, IEEE Transactions on intelligent transportation systems, vol.15,No.4,pp.1690-1705, August 2014.

[2] Amir S. and Mansur V. , 'A new and robust method for character segmentation and recognition in license plate images', Expert Systems with Applications 38 pp.13497-13504, 2011.

[3] C.-N. E. Anagnostopoulos, I. E. Anagnostopoulos, V. Loumos, and E.Kayafas, 'A license plate-recognition algorithm for intelligent transportation system applications’, IEEE Trans. Intell. Trans. Syst., vol. 7, no. 3, pp. 377-392, 2006.

[4] Fikriye O. and Figen O., 'A New License Plate Recognition System Based on Probabilistic Neural Networks', Procedia Technology 1 pp.124 - 128, 2012.

[5] G. A. Samra and F. Khalefah, ' Localization of License Plate Number Using Dynamic Image Processing Techniques and Genetic Algorithms', IEEE Transactions On Evolutionary Computation, Vol. 18, No.2, pp.244-257, April 2014.

[6] Gee-Sern H., Jiun-Chang C. and Yu-Zu C. , 'Application-Oriented License Plate Recognition', IEEE Transactions on vehicular technology”, Vol. 62, No.2, pp.552-561,February 2013.

[7] Shih J.Y., Chian C. H., Jian Y. C. and Chuan Y.C.(2012), 'Practical Homography-Based Perspective Correction Method for License plate Recognition’, IEEE Trans. Veh. Tech., Vol. 12, No.2, pp.198-201.

[8] Wengang Z., Houqiang L., Yijuan L. and Qi T., 'Principal Visual Word Discovery for Automatic License Plate Detection', IEEE transactions on image processing, Vol. 21, No. 9, pp.42-69, September 2012.

[9] Rami A.H. and Khalid A.,' License plate localization using a statistical analysis of Discrete Fourier Transform signal', Computers and Electrical Engineering 40 pp.982-992, 2014.

[10] Runmin W., Nong S., Rui H. and Yuehuan W., 'License plate detection using gradient information and cascade detectors', Optik125 pp.186- 190, 2014. 